\title{
On the Capacity of Disjointly Shared Networks
}

\author{
J. C. Lagarias \\ A. M. Odlyzko \\ D. B. Zagier* \\ AT\&T Labs - Research \\ Murray Hill, NJ 07974
}

\section{Introduction}

Multi-access broadcast channels (such as ETHERNET or Fasnet [5], [6]) have the feature that a message is broadcast to all users, so that only one user can transmit on the channel at a time. The utilization of such a channel would be increased if it were possible for more than one user to occupy the channel at the same time. We consider the performance of a hypothetical channel having the property that users can simultaneously transmit without interference on physically disjoint parts of the channel. We call a channel (or network) having this property a disjointly shared channel. The problem motivating this study is: how much extra capacity does a disjointly shared channel have compared to a multi-access broadcast channel? Here we measure capacity in terms of the number of pairs of users simultaneously using the channel.

We model a disjointly shared channel schematically as a graph, as in Figure 1. The vertices of the graph represent sites of origin or destination of transmissions and the edges are the channel transmission facilities. We model the disjointly shared property of the channel by supposing that when two users are transmitting to each other they seize all vertices and edges on a path connecting the two users, and no other users have access to these vertices or edges during their transmission. (Another model, which we do not consider, supposes that the two users seize the edges on a path connecting the two users, but other user pairs do have access to the vertices on the path. This alternate model corresponds to a situation where the vertices can perform a switching function.) If the graph is a tree, as it is in both examples in Figure 1, the choice of connecting path is unique, but it need not be in other network topologies, such as the graph in Figure 2 .

\footnotetext{
* Permanent addresses: University of Maryland, College Park, MD 20742 and Max-Planck-Institut für Mathematik, Bonn, Fed. Rep. of Germany.
} 
The problem of determining the extra channel capacity possessed by disjointly shared channels compared to broadcast channels does not have a simple answer. The amount of increase in channel capacity depends on the topology of the network and on the probability distribution of origin-destination pairs of the traffic offered to the network.

That network topology has an effect is clear. In the star network of Figure 1, at most one pair of users can transmit at a time, while on the linear network of Figure 1 four pairs of users can simultaneously transmit, namely user 1 with 2,3 with 4,5 with 6 , and 7 with 8 . Consequently, the star disjointly shared network of Figure 1 performs no better than a broadcast network with the same topology. It is clear that the distribution of offered traffic also has an effect, by examination of the linear network in Figure 1. Whenever users 1 and 8 communicate they block all other users, while when user 1 communicates with user 2 any other disjoint user pair is free to transmit. Consequently a network with a large traffic load between users 1 and 2 will be better utilized than a network with heavy traffic between users 1 and 8 .

In the remainder of the paper we carry out a more detailed analysis of the effect of the offered traffic pattern on the capacity for a disjointly shared network with a simple network topology consisting of $k$ parallel cables.

\section{INSERT FIGURE 1 ABOUT HERE}

\section{2. $k$-Cable Network Topology: Main Results and Conclusions}

We study in detail a particularly simple network topology that consists of $k$ parallel disjointly shared channels, each one of which has $n$ entry ports, shown schematically in Figure 2. There are $n$ users individually located at $n$ sites, and the user at site $j$ has access to all $k$ channels at the $j$ th entry port of each channel. For ease of reference we will call this network topology a

INSERT FIGURE 2 ABOUT HERE

k-cable network and each channel a cable. This network topology is suggested by analogy with a proposed 
two-cable network for Fasnet [5], [6]. We define the expected load of the channel for a given distribution of offered traffic to be the expected number of pairs of users communicating at any given time. We interpret the expected load as a measure of the message-carrying capacity of the channel for that distribution of offered traffic.

Our object is to obtain upper bounds on the expected load for disjointly shared channels with the $k$-cable network topology. In doing the analysis we make several assumptions that in general can only increase the performance of the disjointly shared channels. First, we assume the network is synchronous, i.e., the channels are slotted into discrete time intervals. Second, we assume all messages are of duration exactly one time interval. Third, we assume that the requests for message transmission are arranged in a queue by order of arrival and that an oracle (or central controller) can instantaneously reorder the requests in the queue by a bounded amount, into whatever ordering will maximize channel usage. Bounds for the performance of such a network give upper bounds for the performance of a network with no central controller and some decentralized method of resolving message conflicts. Fourth, we model a "heavy traffic" situation in which there are always messages available for transmission. We remark that many multiple access network performance analyses exhibit degradation of network capacity in "heavy traffic" situations. This degradation is a consequence of delays in resolving conflicts in scheduling traffic on the network. Our model assumes that all conflicts are instantaneously resolved, so that the "heavy traffic" situation maximizes channel usage in our model. In particular we will obtain upper bounds for the maximum expected load attainable on the network for a given distribution of offered traffic.

We first bound the maximum expected load attainable on the $k$-cable network in terms of the statistics of message requests. We suppose the queue of traffic requests between users is drawn independently from a probability distribution $T$ on user pairs $(i, j)$. Given a pair $\mathbf{t}=(i, j)$ of users we define the distance $d(\mathbf{t})$ between them to be

$$
d(\mathbf{t})=|i-j|
$$

Let $d^{*}=E[d(T)]$, the expected distance between pairs of users from the distribution $T$. Then we have the following bound.

Theorem A. The expected load $E_{k, n}(T)$ for offered traffic with distribution T satisfies 


$$
E_{k, n}(T) \leq \frac{k n}{d^{*}}
$$

where $d^{*}$ is the expected value of $d(t)$, the distance between users in $T$.

To see this, we note that the total distance available at any time on the $k$ cables to be used by messages is $k(2 n-1)$. Now the first $R$ messages in the queue of requests have expected length asymptotic to $d^{*}$ as $R \rightarrow \infty$. Hence they require at least $\frac{d^{*}}{k n}$ time intervals to be transmitted. Since only a reordering of messages by a bounded number $B$ of positions in the queue is allowed, at most $B$ other messages can be transmitted in the reordered queue before the first $R$ messages are sent. Consequently at most $R+B$ messages can be transmitted in the first $\frac{d^{*}}{k n}$ time intervals. Hence

$$
E_{k, n}(T) \leq \frac{R+B}{\frac{d^{*}}{k n}}=\frac{k n}{d^{*}}+\frac{1}{R}\left(\frac{k n B}{d^{*}}\right)
$$

Letting $R \rightarrow \infty$ gives

$$
E_{k, n}(T) \leq \frac{k n}{d^{*}}
$$

the required result.

This proof of Theorem A is actually valid in the more general situation where the message requests are generated by a stochastic process $T$ which is stationary in the wide sense, so that $E\left[\left.\frac{1}{m} \sum_{i=1}^{m} d\left(t_{i}\right)\right|^{\rceil} \rightarrow d^{*}\right.$ as $m \rightarrow \infty$. This models more realistic situations in which correlation occurs between origin-destination pairs of consecutive messages.

We apply the bound of Theorem A to the case of the uniform offered traffic distribution $U$.

Uniform Offered Traffic. All user pairs wish to communicate with equal probability, i.e., $p_{i j}=\frac{2}{n(n-1)}$ for $1 \leq i, j \leq n$.

The following result shows that the maximum expected load attainable on a $k$-cable disjointly shared network is at most three times that possible over the maximum expected load $k$ attainable on a $k$-cable 
network consisting of $k$ broadcast channels.

Theorem B. The expected load $E_{k, n}(U)$ for uniform offered traffic satisfies

$$
E_{k, n}(U) \leq 3 k
$$

This follows from Theorem A together with the observation that the expected value of the length $|i-j|$ between a pair of users drawn uniformly from $[1, n]$ is $(n+1) / 3$.

Theorem A shows that one can only obtain large expected loads for offered traffic distributions $T$ in which $d^{*}$ is small, i.e., distributions in which most messages travel a short distance on the network. We next analyze a traffic pattern on the network where this is so. To describe this traffic pattern, we introduce some terminology. A configuration is an arrangement of the $n$ users in which each user is either not interested in communicating with anyone, or else wishes to communicate with exactly one other user, who in turn wishes to communicate with the first user. Configurations represent the possible demands for service on the network. A configuration of user pairs is $k$-realizable if there is some way to connect all pairs of users in the configuration who wish to communicate on a $k$-cable network without blocking. The channel traffic pattern we consider is given as follows.

k-Realizable Traffic Pattern. All k-realizable configurations are equally likely to occur.

Our reason for studying this traffic pattern is that it represents a situation in which the $k$-cable network would seem to be used most efficiently. We assume for now that there is some distribution of offered traffic and some mechanism for feeding this traffic onto the network that permits the $k$-realizable traffic pattern to be achieved, and discuss afterwards to what extent these assumptions are reasonable.

The main analytical part of the paper (sections 3-5) is devoted to an analysis of the expected load achieved by the $k$-realizable traffic pattern. We first note that a network with $n$ users and $k \geq n / 2$ cables functions like an infinite resource since all configurations are $k$-realizable. Indeed each configuration contains no more than $n / 2$ pairs of users who wish to communicate, and one can then route each one on a separate cable. A $k$-cable network with $k$ less than $\lfloor n / 2\rfloor$ does not act like an infinite resource in that some configurations are not realizable. We show that the $k$-realizable traffic pattern the expected load is much higher than that achieved for the uniform offered traffic model. 
Theorem $C$. The expected load $E_{k, n}$ for the k-realizable traffic pattern satisfies

$$
E_{k, n} \sim \alpha_{k} n
$$

as $n \rightarrow \infty$ with $k$ fixed. Here $\alpha_{k}$ is a positive constant given by

$$
\alpha_{k}=\frac{\theta_{1}^{(k+1)}}{2 \theta_{1}^{(k+1)}+\sqrt{2}},
$$

and $\theta_{1}^{(k)}$ is the largest zero of the $k$ th Hermite polynomial $H_{k}(x)$. The constants $\alpha_{k}$ satisfy

$$
\alpha_{k}=\frac{1}{2}\left(1-\frac{1}{2 \sqrt{k}}+O\left(k^{-5 / 6}\right)\right)
$$

as $k \rightarrow \infty$. The first few values of the $\alpha_{k}$ are $\alpha_{1}=\frac{1}{4}, \quad \alpha_{2}=\frac{1}{2} \frac{\sqrt{3}-}{1+\sqrt{3}}$ and $\alpha_{3}=\frac{1}{2} \frac{\sqrt{3+\sqrt{6}}}{1+\sqrt{3+\sqrt{6}}}$

Numerically $\alpha_{2}=0.31698 \ldots$ and $\alpha_{3}=0.35004 \ldots$. The Hermite polynomials $H_{k}(x)$ are defined in Section 4 .

Theorem $\mathrm{C}$ asserts that for large $n$ on the average a positive fraction $\alpha_{k} n$ of the users are sending or receiving messages on the network in any given time interval. Furthermore the result that $\alpha_{k} \rightarrow \frac{1}{2}$ as $k \rightarrow \infty$ shows that for large $k$ essentially half of the users are involved in a transmission in each time period, either as a sender or a receiver. The result

$$
E_{k, n} \sim \alpha_{k} n
$$

of Theorem $\mathrm{C}$ is an asymptotic one as $n \rightarrow \infty$, but it is already quite a good approximation for small $k$ and $n$, see Table 1 . 


\begin{tabular}{|c|c|c|c|c|c|c|}
\hline$n$ & 4 & 8 & 12 & 16 & 20 & $\begin{array}{l}\text { limit as } \\
n \rightarrow \infty\end{array}$ \\
\hline 1 & 0.25 & 0.25 & 0.25 & 0.25 & 0.25 & 0.25 \\
\hline 2 & 0.3 & $0.316 \ldots$ & $0.316 \ldots$ & $0.316 \ldots$ & $0.316 \ldots$ & $0.316 \ldots$ \\
\hline 3 & 0.3 & $0.343 \ldots$ & $0.349 \ldots$ & $0.350 \ldots$ & $0.350 \ldots$ & $0.350 \ldots$ \\
\hline 4 & 0.3 & $0.348 \ldots$ & $0.366 \ldots$ & $0.369 \ldots$ & $0.370 \ldots$ & $0.370 \ldots$ \\
\hline 5 & 0.3 & $0.348 \ldots$ & $0.371 \ldots$ & $0.381 \ldots$ & $0.383 \ldots$ & $0.384 \ldots$ \\
\hline 6 & 0.3 & $0.348 \ldots$ & $0.372 \ldots$ & $0.386 \ldots$ & $0.392 \ldots$ & $0.394 \ldots$ \\
\hline 7 & 0.3 & $0.348 \ldots$ & $0.372 \ldots$ & $0.387 \ldots$ & $0.396 \ldots$ & $0.402 \ldots$ \\
\hline
\end{tabular}

Table 1. $n^{-1} E_{k, n}$

We now turn to the problem of showing the existence of an offered traffic distribution $T_{n}$ on $n$ users that would permit the $k$-realizable traffic pattern to be obtained. Assuming all traffic gets on the network, the distribution $T_{n}$ is uniquely specified by the probabilities

$$
p_{i j}=\frac{\# k \text {-realizable configurations on } n \text { users containing }(i j)}{\# k \text {-realizable configurations on } n \text { users }} .
$$

This distribution is very heavily peaked towards users close together in the network topology, for Theorem A and Theorem C together imply that

$$
d^{*} \leq \frac{k}{\alpha_{k}}
$$

as $n \rightarrow \infty$. Even for this particular offered traffic distribution, is there a mechanism to achieve the $k$-realizable traffic pattern on this network? Exact attainment of this traffic pattern seems impossible by any physically realizable means. A centralized controller which sorted and batched arriving message requests using bounded delay $B$ could achieve an arbitrarily close approximation to this traffic pattern as $B \rightarrow \infty$. Using a decentralized traffic access protocol (which is the kind of protocol we imagine being used with such a channel) a close approximation to the $k$-realizable traffic pattern is probably not possible. For this reason the results of Theorem $\mathrm{C}$ should be viewed only as upper bounds on the achievable expected load. These upper bounds may not be too unreasonable, because a heuristic analysis (omitted here) suggests to us that there do exist simple decentralized traffic protocols which would attain expected loads at rates $\beta_{k} n$ for very small constants $\beta_{k}$ for offered traffic with a distribution like $T_{n}$ above. For example, one can consider schemes in which transmission from $i$ to $j$ could only be allowed in certain time slots, i.e. the protocol indirectly coordinates the behavior of different users. The construction and performance analysis of such 
protocols are interesting areas for research which we do not consider further here.

Theorem $\mathrm{C}$ is proved in sections 3-6, which are quite technical and may be skipped. The problem is transformed into a combinatorial problem of counting those permutations on $n$ letters having a special form, the $k$-realizable involutions, and of estimating the average value of a statistic attached to such permutations, the number of cycles.

What may we conclude from these results about the capacity of a disjointly shared channel compared to that of a broadcast channel? Theorem B showed that the capacity of a $k$-cable disjointly shared network increased by only a constant over the capacity of $k$ broadcast channels for uniform offered traffic, while the discussion following Theorem $\mathrm{C}$ suggested that enormous expected loads $\alpha_{k} n$ might be attainable for very special traffic distributions peaked toward short-distance traffic. The latter situation shows a huge increase in capacity over the corresponding $k$-cable broadcast network. However this is not a completely fair comparison: for such an offered traffic distribution one could achieve a much greater capacity in a broadcast network by chopping up some of the $k$ cables into smaller disjoint pieces. For example, with $2^{k}$ users and $k$ cables, cut the $j$ th cable into $2^{j}$ segments of length $2^{k-j}$, and have users attempt to transmit messages on the shortest cable connecting them to their intended receiver. The short distance transmissions would then go on the short cable segments. Without making this quantitative, we may say that the relative advantage in capacity of a $k$-cable disjointly shared network over a properly specified broadcast network is much smaller than that suggested by Theorem C, and Theorem A suggests that it is bounded by a small constant for reasonable offered traffic distributions.

Finally we observe that this analysis suggests that, for this theoretical model, and for very special offered traffic patterns, some increase in usage of a multiply-connected broadcast channel network might be achieved by splitting it into several disjoint segments in such a way that it is still possible for any two users to communicate over one segment or another. However this observation seems of little practical importance, because the special traffic patterns needed are unlikely to occur, and because other (crucial) factors omitted in this model favor connected broadcast network topologies. 


\section{3. $k$-Realizable Involutions}

In the remaining sections, we prove Theorem $\mathrm{C}$. We formulate the problems of estimating the expected load for the $k$-realizables traffic distribution in terms of permutations. We use standard terminology for permutations, cf. Berge [2; pp. 110-123]. Let $S_{n}$ denote the set of permutations acting on $\{1, \ldots, n\}$. The permutations we enumerate are the involutions; i.e., those $\sigma \in S_{n}$ with $\sigma^{2}=$ identity. To say this another way, an involution is a permutation $\sigma$ whose cycle structure is a mixture of 1-cycles (fixed points) and 2-cycles. A configuration of $2 n$ users corresponds to an involution on $S_{n}$. We can formulate the notion that a configuration is realizable on a $k$-cable network purely in terms of permutations by introducing the notion of crossing number.

Definition. Let $\sigma \in S_{n}$ be an involution. The crossing number $c_{i}(\sigma)$ of $\sigma$ at $i$, for $1 \leq i \leq n-1$, is

$$
c_{i}(\sigma)=\#\{j: j \leq i<\sigma(j)\} .
$$

If we imagine the elements $1, \ldots, n$ as being arranged in a line, with a wire running between each connected pair of users connected to each other, then $c_{i}(\sigma)$ is the number of wires crossing the interval between $i$ and $i+1$.

Definition. An involution $\sigma$ is $k$-realizable if $c_{i}(\sigma) \leq k$ for all $i$.

The notion of a $k$-realizable permutation corresponds exactly to a configuration realizable on a $k$-cable network.

Our main result estimates the expected number of 2-cycles in a $k$-realizable involution. We do this in Section 6. To carry out that estimation, we first study (in sections 4 and 5) involutions with no fixed point. This of course requires than $n$ be even, say $n=2 N$.

Definition. $i_{k}(N)$ is the number of $k$-realizable involutions $\sigma \in S_{2 N}$ with no fixed points. We set $i_{k}(0)=1$ for $k \geq 0$ and $i_{0}(N)=0$ for $N \geq 1$.

\section{Theorem D. If}




$$
I_{k}(x)=\sum_{N=0}^{\infty} i_{k}(N) x^{N}
$$

then

$$
\begin{aligned}
I_{k}(x)=\frac{1}{1-\frac{x}{1-\frac{2 x}{1-\ddots}}} & \\
& \quad-\frac{(k-1) x}{1-k x}
\end{aligned}
$$

More explicitly,

$$
I_{k}(x)=\frac{R_{k}(x)}{Q_{k}(x)}
$$

where $Q_{0}(x)=1, Q_{1}(x)=1-x, R_{0}(x)=R_{1}(x)=1$, and both $Q_{k}(x)$ and $R_{k}(x)$ satisfy the recurrence

$$
U_{k+1}(x)=U_{k}(x)-(k+1) x U_{k-1}(x) \text { for } k \geq 1
$$

(see Tables 2 and 3). 


\begin{tabular}{c|cccccccc}
\multicolumn{1}{l}{\begin{tabular}{c}
$\mathrm{N}$ \\
\cline { 2 - 8 } 0
\end{tabular}} & 0 & 1 & 2 & 3 & 4 & 5 & 6 & 7 \\
\cline { 2 - 8 } 1 & 1 & 0 & 0 & 0 & 0 & 0 & 0 & 0 \\
2 & 1 & 1 & 1 & 1 & 1 & 1 & 1 & 1 \\
3 & 1 & 1 & 3 & 9 & 27 & 81 & 243 & 729 \\
4 & 1 & 1 & 3 & 15 & 105 & 825 & 6675 & 54375 \\
5 & 1 & 1 & 3 & 15 & 105 & 945 & 9675 & 104175 \\
6 & 1 & 1 & 3 & 15 & 105 & 945 & 10395 & 130095 \\
7 & 1 & 1 & 3 & 15 & 105 & 945 & 10395 & 135135
\end{tabular}

Table 2. $i_{k}(N)$

\begin{tabular}{lll}
$\underline{k}$ & \multicolumn{1}{c}{$\underline{Q_{k}(x)}$} & \multicolumn{1}{c}{$\underline{R_{k}(x)}$} \\
0 & 1 & 1 \\
1 & $1-x$ & 1 \\
2 & $1-3 x$ & $1-2 x$ \\
3 & $1-6 x+3 x^{2}$ & $1-5 x$ \\
4 & $1-10 x+15 x^{2}$ & $1-9 x+8 x^{2}$ \\
5 & $1-15 x+45 x^{2}-15 x^{3}$ & $1-14 x+33 x^{2}$
\end{tabular}

Table 3. $\quad Q_{k}(x)$ and $R_{k}(x)$

Since the total number of $k$-realizable involutions $\sigma \in S_{n}$ is

$$
\sum_{N=0}^{[n / 2]}\left(\begin{array}{c}
n \\
2 N
\end{array}\right) i_{k}(N)
$$

this yields an enumeration of all $k$-realizable involutions.

P. Flajolet has pointed out that he previously obtained Theorem D as a corollary to very general permutation enumeration results $[4$, Theorem $5 \mathrm{~B}]$.

Our goal is to prove Theorem C, which we reformulate in terms of permutations. Let $E_{k}(n)$ be the average number of 2-cycles in all $k$-admissible involutions of $S_{n}$. Using this notation the expected load 
$E_{k, n}$ of the $k$-admissible message distribution as a $k$-cable disjointly shared network with $n$ users is just $E_{k}(n)$. Theorem $\mathrm{C}$ is an immediate consequence of the following result concerning $k$-admissible involutions.

Theorem E. For an arbitrary $n$, even or odd, the average $E_{k}(n)$ of the number of 2-cycles among all $k$ admissible involutions $\sigma \in S_{n}$ satisfies

$$
\lim _{n \rightarrow \infty} \frac{E_{k}(n)}{n}=\alpha_{k}
$$

where

$$
\alpha_{k}=\frac{\theta_{1}^{(k+1)}}{2 \theta_{1}^{(k+1)}+\sqrt{2}},
$$

and $\theta_{1}^{(k)}$ is the largest zero of the Hermite polynomial $H_{k}(x)$. As a consequence, as $k \rightarrow \infty$

$$
\alpha_{k}=\frac{1}{2}\left(1-\frac{1}{2 \sqrt{k}}+O\left(k^{-5 / 6}\right)\right)
$$

The first few values of the $\alpha_{k}$ are $\alpha_{1}=\frac{1}{4}, \alpha_{2}=\frac{1}{2} \frac{\sqrt{3}-}{1+\sqrt{3}}$ and $\alpha_{3}=\frac{1}{2} \frac{\sqrt{3+\sqrt{6}}}{1+\sqrt{3+\sqrt{6}}}$.

We prove Theorem D in the next section, derive results about Hermite polynomials in Section 5 and finally prove Theorem E in Section 6.

We remark that $i_{k}(N)$ stabilizes for $k$ large (cf. Table 2). Indeed if $k \geq N$, then there is no restriction on the crossing number, and $i_{k}(N)$ counts all involutions with no fixed points. To count them, note that there are $2 N-1$ choices for an element to pair 1 with. The next unpaired user has $2 N-3$ choices, and so on, so

$$
i_{k}(N)=(2 N-1) ! !=\frac{(2 N) !}{2^{N} \cdot N !}=1 \cdot 3 \cdot 5 \cdot 7 \cdot \ldots \cdot(2 N-1)
$$

for $k \geq N$. Hence, letting $k \rightarrow \infty$ in Theorem $\mathrm{D}$ we obtain:

Theorem $F$. The following is a formal power series identity: 


$$
\frac{1}{1-\frac{x}{1-\frac{2 x}{1-\frac{3 x}{1-\ldots}}}}=\sum_{n=0}^{\infty}(2 N-1) ! ! x^{N} .
$$

We remark that this theorem can also be proved directly using standard methods in the analytic theory of continued fractions. Indeed we have

$$
\frac{1}{1+\frac{x}{1+\frac{2 x}{1+\ldots}}}=\int_{0}^{\infty} e^{-t-\frac{x}{2} t^{2}} d t
$$

valid for $\operatorname{Re}(x) \geq 0$. (See [8, p.353] for analogous results. Take $\alpha=1 / 2, x=2 z$ in (92.4).) If we differentiate the integral on the right $n$ times with respect to $x$, and set $x=0$, we obtain

$$
\begin{aligned}
& (-1)^{n} x^{n} 2^{-n} \int_{0}^{\infty} t^{2 n} e^{-t} d t \\
& =(-1)^{n} x^{n} 2^{-n}(2 n) !=(-1)^{n} \cdot n !(2 n-1) ! ! x^{n},
\end{aligned}
$$

which yields Theorem F.

\section{Proof of Theorem D}

Associate to each fixed-point-free involution $\sigma \in S_{2 N}$ its vector $\mathbf{v}(\sigma)$ of crossing numbers:

$$
\mathbf{v}(\sigma)=\left(c_{1}(\sigma), c_{2}(\sigma), \ldots, c_{2 N-1}(\sigma)\right) .
$$

Under what circumstances can a vector

$$
\mathbf{v}=\left(c_{1}, \ldots, c_{2 N-1}\right)
$$

arise as the crossing number vector $\mathbf{v}(\sigma)$ of some fixed-point-free involution $\sigma$ ? We next show that necessary and sufficient conditions are given by

$$
\begin{aligned}
& \text { (i) } c_{1}=c_{2 N-1}=1, \\
& \text { (ii) } c_{i} \geq 0 \text { for } 2 \leq i \leq 2 N-2, \\
& \text { (iii) } c_{i+1}=c_{i} \pm 1 \text { for } 2 \leq i \leq 2 N-2 .
\end{aligned}
$$


These conditions are clearly necessary. (i) holds since both user 1 and user $N$ are talking to someone. (ii) is obvious. (iii) holds since $c_{i+1}=c_{i}+1$ if user $i+1$ is talking to user $j$ with $j>i+1$ and $c_{i+1}=c_{i}-1$ if $j \leq i$. These conditions are also sufficient. We can construct all the complete involutions with a given pattern (4.2) that satisfies (4.3) as follows. We induct on $\underline{i}$, for $1 \leq i \leq 2 N-2$. Whenever $c_{i+1}=c_{i}+1$, we have user $i+1$ connect to person $j$ with $j>i+1, j$ to be selected later. We say user $i+1$ calls to his right. Whenever $c_{i+1}=c_{i}-1$, we connect user $i+1$ to any $j<i$ such that person $j$ was connected to his right but has not yet been connected to anyone in particular. (There is at least one such user since $c_{i} \geq 1$ is forced by $c_{i+1} \geq 0$.) Hence this construction can always be completed, and in fact in exactly

$$
N(\mathbf{v})=\prod_{\substack{i \\ c_{i+1}<c_{i}}} c_{i}
$$

distinct ways.

The conditions (4.3) can be viewed as those of a random walk taking steps $s_{i}= \pm 1$ with $c_{0}=c_{2 m}=0$ with the condition that at all intermediate steps the cumulative sums satisfy

$$
c_{i}=s_{1}+s_{2}+\ldots+s_{i} \geq 0
$$

We will count fixed-point-free involutions by first introducing an auxiliary counting function for which we can derive a recurrence.

Definition. Let $C_{k, t, N}$ denote the set of fixed-point-free involutions on $2 N$ points with crossing vectors $\mathbf{v}=\left(c_{1}, \ldots, c_{2 N-1}\right)$ which satisfy

1. All $c_{i} \leq k$,

2. For exactly $t$ distinct $i, c_{i}=k$,

3. If $t=0$, then some $c_{i}=k-1$.

Let $f_{k, t}(N)$ denote the cardinality of $C_{k, t, N}$.

We show $f_{k, t}(N)$ satisfies the following recursion.

Lemma 4.1. For all $t \geq 0$, 


$$
f_{k, t}(N)=\sum_{s \geq 1} f_{k-1, s}(N-t) k^{t}\left(\begin{array}{c}
t+s-1 \\
t
\end{array}\right)
$$

Proof. View the crossing number vector $\mathbf{v}$ of a fixed-point-free involution counted in $f_{k, t}(N)$ as a walk. We say that $\mathbf{v}$ has a run of $r$ values of $k$ if there is an $i$ with

$$
c_{i}=c_{i+2}=\ldots=c_{i+2(r-1)}=k \text {. }
$$

As an example, the crossing number vector in Figure 3, with $k=3$, has a run of length 2 starting at $i=3$, and a run of length 1 starting at $i=9$. If $r_{1}, \ldots, r_{\nexists \rightarrow}$ denote the lengths of maximal runs in $\mathbf{v}$ then

$$
r_{1}+\ldots+r_{\equiv+}=t
$$

We form a new crossing number vector $\mathbf{v}^{*}$ with $N^{*}=N-t$ as follows. For each maximal run from $c_{i}$ to $c_{i+2(r-1)}$ we delete $c_{i}$ through $c_{i+2 r-1}$. Using the random walk characterization of crossing number vectors, we conclude that the new vector $\mathbf{v}$ * resulting from this operation is a crossing number vector. As an example, Figure 4 shows the new crossing number vector $\mathbf{v}^{*}$ derived from $\mathbf{v}$ in Figure 1 . If $t=0$, then $\mathbf{v} *$ is simply taken to be $\mathbf{v}$.

INSERT FIGURE 3 ABOUT HERE

\section{INSERT FIGURE 4 ABOUT HERE}

Note that the new vector $\mathbf{v}^{*}$ contains at least one crossing number equal to $k-1$. Hence $\mathbf{v}^{*}$ is in $C_{k-1, s, N-t}$ for some $s \geq 1$.

We can reverse this operation. Let $\mathbf{v}^{*}$ be in $C_{k-1, s, N-t}$. In how many ways can we obtain a crossing number vector of an element in $C_{k, t, N}$ ? At each of the $s$ peaks of $\mathbf{v}^{*}$ where $c_{i}^{*}=k-1$, we can insert a run of $r_{i}$ peaks with crossing number $k$. In order to obtain an element of $C_{k, t, N}$ it is necessary and sufficient that

$$
r_{1}+r_{2}+\ldots+r_{s}=t
$$


The number of ways to choose a vector of nonnegative integers $\left(r_{1}, \ldots, r_{s}\right)$ satisfying $(4.7)$ is $\left(\begin{array}{c}t+s-1 \\ s-1\end{array}\right)$.

Now let $\mathbf{v} *$ be the crossing number vector of a $\sigma \in C_{k-1, s, N-t}$. This vector arises from

$$
N(\mathbf{v} *)=\prod_{\substack{i \\ c_{i+1}^{*}<c_{i}^{*}}} c_{i}^{*}
$$

different complete involutions. If $\mathbf{v}$ is a crossing number vector associated to $C_{k, t, N}$ derived from $\mathbf{v} *$ then

$$
\begin{aligned}
N(\mathbf{v}) & =\prod_{\substack{i \\
c_{i+1}<c_{i}}} c_{i}=k^{t}{\underset{c}{c_{i+1}}{ }^{*}<c_{i}}_{\prod_{i}} c_{i}^{*} \\
& =k^{t} N\left(\mathbf{v}^{*}\right) .
\end{aligned}
$$

since the vector $\mathbf{v}$ is the same as $\mathbf{v}^{*}$ except that $t$ occurrences of the crossing number pattern $(k, k-1)$ have been added. Consequently the $f_{k-1, s}(N-t)$ elements of $C_{k-1, s, N-t}$ yield

$$
k^{t}\left(\begin{array}{c}
t+s-1 \\
s-1
\end{array}\right) f_{k-1, s}(N-t)
$$

distinct elements of $C_{k, t, N}$ under the reverse correspondence.

The lemma follows by summing over $s \geq 1$. (Note that for $s \geq N$ we have $f_{k, s}(N)=0$.)

Note. We can always include $s=0$ in the summation (4.6), since $\left(\begin{array}{c}t-1 \\ t\end{array}\right)=0$.

Next we introduce the generating function

$$
F_{k, t}(x)=\sum_{N=0}^{\infty} f_{k, t}(N) x^{N}
$$

where we make the convention that $f_{1,0}(0)=1$ and $f_{k, t}(0)=0$ otherwise.

Note that $f_{k+1,0}(N)=i_{k}(N)-i_{k-1}(N)$, so that

$$
F_{k+1,0}(x)=I_{k}(x)-I_{k-1}(x)
$$

The recursion of Lemma 4.1 implies that $F_{k, t}(x)$ satisfies 


$$
F_{k, t}(x)=k^{t} x^{t} \sum_{s=0}^{\infty}\left\{\begin{array}{c}
t+s-1 \\
t
\end{array}\right\} F_{k-1, s}(x)
$$

Direct evaluation of $f_{1, t}(N)$ leads to the initial conditions:

$$
F_{1, t}(x)=x^{t}, \quad 0 \leq t<\infty .
$$

Using the recurrence (4.11) we then calculate that

$$
F_{2, t}(x)=\frac{x}{1-x}\left(\frac{2 x}{1-x}\right)^{t}, \quad 0 \leq t<\infty .
$$

This suggests we try to obtain a solution of the recursion (4.11) of the form

$$
F_{k, t}(x)=A_{k}(x) B_{k}(x)^{t}
$$

where $A_{k}(x)$ and $B_{k}(x)$ are formal power series. Substituting (4.13) into (4.11) and using the binomial theorem we find that

$$
A_{k} B_{k}^{t}=(k x)^{t} \sum_{s=1}^{\infty}\left(\begin{array}{c}
t+s-1 \\
t
\end{array}\right) A_{k-1} B_{k-1}^{s}=(k x)^{t} A_{k-1} B_{k-1}\left(1-B_{k-1}\right)^{-t-1} .
$$

Hence (4.13) will be a solution of (4.11) if the functions $A_{k}(x), B_{k}(x)$ satisfy the recursions

$$
\begin{aligned}
& A_{k}(x)=A_{k-1}(x) \frac{B_{k-1}(x)}{1-B_{k-1}(x)}, \\
& B_{k}(x)=\frac{k x}{1-B_{k-1}(x)},
\end{aligned}
$$

together with the initial conditions

$$
\begin{aligned}
& A_{1}(x)=1, \\
& B_{1}(x)=x,
\end{aligned}
$$

implied by (4.12).

The recursion (4.15) with (4.17) implies that

$$
A_{k+1}(x)=\prod_{i=1}^{k} \frac{B_{i}(x)}{1-B_{i}(x)}
$$

while (4.16) yields 


$$
\begin{aligned}
& B_{k}(x)=\frac{k x}{1-\frac{(k-1) x}{1-\ddots}} \\
&-\frac{2 x}{1-x}
\end{aligned}
$$

We now set

$$
B_{k+1}(x)=\frac{P_{k}(x)}{Q_{k}(x)},
$$

where $P_{k}(x), Q_{k}(x)$ are relatively prime polynomials with $P_{k}(0)=0, Q_{k}(0)=1$. Then (4.16) gives

$$
\frac{P_{k}(x)}{Q_{k}(x)}=\frac{(k+1) x Q_{k-1}(x)}{Q_{k-1}(x)-P_{k-1}(x)},
$$

so that

$$
\begin{gathered}
P_{k}(x)=(k+1) x Q_{k-1}(x), \\
Q_{k}(x)=Q_{k-1}(x)-P_{k-1}(x) .
\end{gathered}
$$

This implies that $Q_{k}(x)$ satisfies the recurrence

$$
\begin{aligned}
Q_{k}(x) & =Q_{k-1}(x)-k x Q_{k-2}(x), \\
Q_{-1}(x) & =Q_{0}(x)=1,
\end{aligned}
$$

i.e. these are the polynomials $Q_{k}$ defined in Theorem A. Next substitute (4.22) into (4.21) to get

$$
B_{k}(x)=k x \frac{Q_{k-2}(x)}{Q_{k-1}(x)} .
$$

This formula and (4.24) yield

$$
1-B_{k}(x)=\frac{Q_{k}(x)}{Q_{k-1}(x)},
$$

so that

$$
\frac{B_{k}(x)}{1-B_{k}(x)}=k x \frac{Q_{k-2}(x)}{Q_{k}(x)} .
$$

Thus, using (4.27), the product (4.19) telescopes to yield 


$$
A_{k+1}(x)=k ! x^{k} \frac{1}{Q_{k}(x) Q_{k-1}(x)} .
$$

Now we introduce polynomials $R_{k}(x)$ with

$$
R_{0}(x)=1, R_{1}(x)=1,
$$

and which satisfy the recurrence (4.24) for $k \geq 2$. Then

$$
\left[\begin{array}{ll}
R_{0} & R_{1} \\
Q_{0} & Q_{1}
\end{array}\right]=\left[\begin{array}{cc}
1 & 1 \\
1 & 1-x
\end{array}\right] .
$$

The recurrence (4.24) gives

$$
\left[\begin{array}{ll}
R_{k-1} & R_{k} \\
Q_{k-1} & Q_{k}
\end{array}\right]=\left[\begin{array}{ll}
R_{k-2} & R_{k-1} \\
Q_{k-2} & Q_{k-1}
\end{array}\right]\left[\begin{array}{cc}
0 & -k x \\
1 & 1
\end{array}\right] .
$$

Hence

$$
\left.\left.\left.\left[\begin{array}{ll}
R_{k-1} & R_{k} \\
Q_{k-1} & Q_{k}
\end{array}\right]=\left[\begin{array}{cc}
0 & 1
\end{array}\right]\left[\begin{array}{cc}
0 & -x \\
1 & 1
\end{array}\right] \mid \begin{array}{cc}
1 & 1
\end{array}\right] \mid \begin{array}{cc}
0 & -2 x \\
1 & 1
\end{array}\right] \ldots \mid \begin{array}{cc}
0 & -k x \\
1 & 1
\end{array}\right] .
$$

Taking determinants of both sides, we get

$$
R_{k}(x) Q_{k-1}(x)-R_{k-1}(x) Q_{k}(x)=k ! x^{k} .
$$

Then (4.28) and (4.30) yield

$$
A_{k+1}(x)=\frac{R_{k}(x)}{Q_{k}(x)}-\frac{R_{k-1}(x)}{Q_{k-1}(x)} .
$$

We also have

$$
A_{k+1}(x)=F_{k+1,0}(x)=I_{k}(x)-I_{k-1}(x) .
$$

But now (4.32) gives us a sum for $I_{k}(x)$ in terms of the $A_{m}(x)$, which by (4.31) telescopes to yield

$$
I_{k}(x)=\frac{R_{k}(x)}{Q_{k}(x)} .
$$

Now (4.29) implies that 


$$
\begin{aligned}
& \frac{R_{k}(x)}{Q_{k}(x)}=\frac{1}{1-\frac{x}{1-\frac{2 x}{\ddots}}} \\
& 1-\frac{(k-1) x}{1-k x} .
\end{aligned}
$$

This completes the proof of Theorem D.

\section{Hermite polynomials and Zeros}

The polynomials $Q_{k}(x)$ are related to the Hermite polynomials.

Lemma 5.1. $Q_{k}(x)=\left(\sqrt{ } \frac{x}{2}\right)^{j^{k+1}} H_{k+1}\left(\frac{1}{\sqrt{2 x}}\right)$, where $H_{k}(x)$ is the $k^{\text {th }}$ Hermite polynomial.

Proof. The Hermite polynomials satisfy $H_{1}(x)=2 x, H_{2}(x)=4 x^{2}-2$, so that the Lemma holds for $k=0,1$. Furthermore, we have $[1,7]$

$$
H_{k+1}(x)=2 x H_{k}(x)-2 k H_{k-1}(x),
$$

which in view of the recurrence (4.24) for the $Q_{k}(x)$ yields an immediate proof of the Lemma by induction.

For our application we need to know the size of the smallest zero of $Q_{k}(x)$. By Lemma 5.1 this means we need information about the largest zero of $H_{k}(x)$. We recall the following theorem [7, Thm. 6.32].

Proposition 5.2. All the zeros of $H_{k}(x)$ are real and positive. The largest zero $\theta_{1}^{(k)}$ of $H_{k}(x)$ satisfies

$$
\theta_{1}^{(k)}<(2 k+1)^{1 / 2}-c_{0}(2 k+1)^{1 / 6},
$$

with

$$
c_{0}=\frac{1}{3 \sqrt{6}} i_{o}=1.85575 \ldots
$$

where $i_{0}$ is the smallest zero of Airy's function Ai(x). Furthermore 


$$
\theta_{1}^{(k)}=(2 k+1)^{1 / 2}-\left(c_{0}+\varepsilon(k)\right)(2 k+1)^{1 / 6}
$$

where $\varepsilon(k) \rightarrow 0$ as $k \rightarrow \infty$.

We now estimate $i_{k}(N)$ as $N \rightarrow \infty$, with $k$ fixed.

Lemma 5.3. We have

$$
\lim _{N \rightarrow \infty} \frac{1}{N} \log \left(i_{k}(N)\right)=\log 2\left(\theta_{1}^{(k+1)}\right)^{2} .
$$

Proof. Using the formula for $I_{k}(x)$ given in Theorem C, and expanding in partial fractions, we have

$$
I_{k}(x)=d_{0}^{(k)}-\sum_{i=1}^{\nexists+} \frac{c_{i}^{(k)} \phi_{i}^{(k)}}{x-\phi_{i}^{(k)}}
$$

where $\phi_{i}^{(k)}$ are the roots of $Q_{k}(x)$, which are positive real and distinct using Lemma 5.1 and Proposition 5.2. We suppose $\phi_{1}^{(k)}$ is the smallest root. Now (5.5) gives for $N \geq 1$ that

$$
i_{k}(N)=\sum_{i=1}^{\nexists} c_{i}^{(k)}\left[\phi_{i}^{(k)}\right]^{-N}
$$

Note $c_{1}^{(k)} \neq 0$ since $\left(Q_{k}(x), R_{k}(x)\right)=1$ by $(4.30)$. Hence

$$
\lim _{N \rightarrow \infty} \frac{1}{N} \log \left(i_{k}(N)\right)=-\log \phi_{1}^{(k)} .
$$

Using Lemma 5.1, and Proposition 5.2, we have

$$
\frac{1}{\sqrt{2 \overline{\phi_{1}^{(k)}}}}=\theta_{1}^{(k+1)}
$$

so that

$$
\left[\phi_{1}^{(k)}\right]^{-1}=2\left(\theta_{1}^{(k+1)}\right)^{2}
$$

which proves the lemma.

\section{Mean value of the number of user pairs.}

Proof of Theorem E. For an arbitrary $n$, the total number $T_{k}(n)$ of $k$-realizable configurations between 
$n$ users is given by

$$
T_{k}(n)=\sum_{N=0}^{[n / 2]}\left(\begin{array}{c}
n \\
2 N
\end{array}\right) i_{k}(N)
$$

since there are $\left(\begin{array}{c}n \\ 2 N\end{array}\right)$ ways of picking $2 N$ users and $i_{k}(N)$ ways of connecting them completely. Using (5.6), and (5.7), this is

$$
\begin{aligned}
T_{k}(n)= & \sum_{N=0}^{[n / 2]}\left(\begin{array}{c}
n \\
2 N
\end{array}\right) c_{1}^{(k)} 2^{N}\left(\theta_{1}^{(k+1)}\right)^{2 N} \\
& +O\left(\sum_{N=0}^{[n / 2]}\left(\begin{array}{c}
n \\
2 N
\end{array}\right) 2^{N}\left(\theta_{2}^{(k+1)}\right)^{2 N}\right),
\end{aligned}
$$

where the $O$-constant depends on $k$. Since

$$
\sum_{N=0}^{[n / 2]}\left(\begin{array}{c}
n \\
2 N
\end{array}\right) x^{N}=\frac{1}{2}\left\{\left(1+\sqrt{ } x \bar{s}^{n}+\left(1-\sqrt{x} \overline{)^{n}}\right\}\right\},\right.
$$

we obtain

$$
T_{k}(n)=\frac{1}{2} c_{1}^{(k)}\left(1+\sqrt{ } 2 \bar{\theta}_{1}^{(k+1)}\right)^{n}+O\left(\phi^{n}\right)
$$

where $\phi=\max \left(\left|1-\sqrt{2} \bar{\theta}_{1}^{(k+1)}\right| 1+\sqrt{2} \bar{\theta}_{2}^{(k+1)}\right)$ is smaller than $1+\sqrt{2} \bar{\theta}_{1}^{(k+1)}$.

On the other hand, the total number of connections in all these configurations is

$$
U_{k}(n)=\sum_{N=0}^{[n / 2]} N\left(\begin{array}{c}
n \\
2 N
\end{array}\right) i_{k}(N)
$$

Proceeding as above we obtain

$$
U_{k}(n)=\sum_{N=0}^{[n / 2]} N\left(\begin{array}{c}
n \\
2 N
\end{array}\right) c_{1}^{(k)} 2^{N}\left(\theta_{1}^{(k+1)}\right)^{2 N}+O\left(n \phi^{n}\right)
$$

and

$$
\begin{aligned}
\sum_{N=0}^{[n / 2]} N\left(\begin{array}{c}
n \\
2 N
\end{array}\right) x^{N} & =\frac{1}{2} x \frac{d}{d x}\left(\left(1+\sqrt{ } x \bar{s}^{n}+(1-\sqrt{ } x)^{n}\right)\right. \\
& =\frac{1}{4} n \sqrt{ } x \overline{(} 1+\sqrt{ } x \bar{x}^{n-1}+O\left(n \sqrt{ } x \overline{\mid} 1-\sqrt{ } x \overline{\mid}^{n-1}\right)
\end{aligned}
$$


Hence

$$
U_{k}(n)=\frac{1}{4} n c_{1}^{(k)} \sqrt{2} \bar{\theta}_{1}^{(k+1)}\left(1+\sqrt{ } 2 \bar{\theta}_{1}^{(k+1)}\right)^{n-1}+O\left(n \phi^{n}\right) .
$$

Now

$$
E_{k}(n)=\frac{U_{k}(n)}{T_{k}(n)}
$$

Combining (6.4) and (6.6), we get

$$
\lim _{N \rightarrow \infty} \frac{E_{k}(n)}{n}=\frac{1}{2} \frac{\sqrt{ } 2 \bar{\theta}_{1}^{(k+1)}}{1+\sqrt{ } 2 \bar{\theta}_{1}^{(k+1)}} .
$$

Next, using (5.7) to estimate $\theta_{1}^{(k+1)}$, the asymptotic estimate for $\alpha_{k}$ follows.

\section{Remarks.}

1) Since

$$
\begin{aligned}
& Q_{1}(x)=1-x, \quad \phi_{1}^{(1)}=1, \quad\left(\phi_{1}^{(1)}\right)^{-1 / 2}=1, \\
& Q_{2}(x)=1-3 x, \quad \phi_{1}^{(2)}=1 / 3, \quad\left(\phi_{1}^{(2)}\right)^{-1 / 2}=\sqrt{3}- \\
& Q_{3}(x)=1-6 x+3 x^{2}, \quad \phi_{1}^{(3)}=1-\frac{\sqrt{6}}{3}, \quad\left(\phi_{1}^{(3)}\right)^{-1 / 2}=\sqrt{3+\sqrt{6}},
\end{aligned}
$$

we have

i) $\quad \alpha_{1}=\frac{1}{4}$. (In fact $E_{1}(n)=\frac{1}{4} n$ for all $\left.n\right)$.

ii) $\alpha_{2}=\frac{1}{2} \frac{\sqrt{3}-}{1+\sqrt{3}}$

iii) $\alpha_{3}=\frac{1}{2} \frac{\sqrt{3+\sqrt{6}}}{1+\sqrt{3+\sqrt{6}}}$.

2) We can give exact formulae for $T_{k}(n), U_{k}(n), E_{k}(n)$ for small $k$ using the partial fraction expansion (5.10) along with formulae (6.3), (6.6). 


\section{REFERENCES}

1. M. Abramowitz and I. A. Stegun, eds., Handbook of Mathematical Functions, National Bureau of Standards, 9th. printing, 1970.

2. C. Berge, Principles of Combinatorics, New York: Academic Press, 1971.

3. L. Comtet, Advanced Combinatorics, Reidel, 1974

4. P. Flajolet, Combinatorial aspects of continued fractions, Discrete Math. 32 (1980), 125-161.

5. J. O. Limb, FASNET: Proposal for a High Speed Local Network, Proc. 2nd International Office Information Systems Workshop, Saint Maximum, France, Oct. 13-15, 1981.

6. J. O. Limb and C. Flores, Description of Fasnet, a Unidirectional Local Area Network, Bell System Tech. J. 62 (1982), 1413-1440.

7. G. Szegö, Orthogonal Polynomials, Am. Math. Society, 3rd ed., 1967.

8. C. T. C. Wall, Analytic Theory of Continued Fractions, Chelsea reprint, 1973. 


\title{
On the Capacity of Disjointly Shared Networks
}

\author{
J. C. Lagarias \\ A. M. Odlyzko \\ D. B. Zagier*
}

AT\&T Labs - Research

Murray Hill, NJ 07974

ABSTRACT

Multi-access broadcast channels have the property that only one user can successfully transmit on the channel at a time. We consider a hypothetical channel called a disjointly shared channel in which more than one user pair can communicate simultaneously over physically disjoint parts of the channel. We consider the question of how much extra capacity such a channel has over that of a broadcast channel, as measured by the number of user pairs on the channel. The amount of extra capacity depends on the topology of the channel and the distribution of offered traffic. We analyze the problem for a particular disjointly shared channel having $n$ users whose topology consists of $k$ disjoint parallel cables. For an offered traffic pattern equally weighting each pair of users the capacity increases by at most a factor of three over that of $k$ disjoint multi-access broadcast channels, i.e. on average at most $3 k$ pairs of users will be communicating. For a specific offered traffic pattern which heavily weights connections between users close to each other the capacity is approximately $\alpha_{k} n$ for a constant $\alpha_{k}$ depending on the number of cables $k$, and it is shown that $1 / 4 \leq \alpha_{k}<1 / 2$ with $\alpha_{k}$ increasing to $1 / 2$ as $k \rightarrow \infty$. The analysis for the second traffic pattern leads to a permutation enumeration problem which is solved using generating functions, continued fractions and Hermite polynomials.

Keywords: multi-access channel, capacity, disjointly shared channel

\footnotetext{
* Permanent addresses: University of Maryland, College Park, MD 20742 and Max-Planck-Institut für Mathematik, Bonn, Fed. Rep. of Germany.
} 